\title{
Shared pain: Lessons from the labour room in Istanbul
}

\author{
Patricia K Morley-Forster MD
}

$\mathrm{T}$ he current issue of Pain Research $\mathcal{E}$ Management contains an article by Yildirim and Sahin (pages 183-187) from the Faculty of Nursing at the University of Istanbul (Istanbul, Turkey). At first glance, this paper appears to be of interest only to those involved in the day-to-day care of women in labour, but a closer look reveals significant lessons for those dedicated to the improvement of pain management globally.

This randomized clinical trial, conducted in 40 low-risk pregnant women at the SSK Bakirkoy Women and Children's Hospital (Istanbul, Turkey), demonstrated that education about nonpharmacological methods of pain relief, continuous nursing support and massage techniques significantly reduced both the severity of pain and the emotional distress of labour for women. In addition, postpartum feelings about the labour and delivery room experience were far more positive in the experimental group than in the control group (65\% versus $20 \%$ ). From a North American standpoint, it might have been easy to dismiss this study as irrelevant to our practice. For one, the described 'interventions' of prenatal education and intrapartum nursing support are considered to be a standard part of obstetric care in even the smallest birthing centre in Canada. Weaknesses in the study design included a small sample size (40 patients), nonrandom selection of volunteers, lack of blinding and failure to quantify the relative importance of education versus touch and massage. Yet, the authors deserve credit for even undertaking this research in a culture that has traditionally undervalued treatment of pain in women, and for doing it in a credible fashion. Some of the lessons from this study have the potential to extend well beyond the walls of the SSK Bakirkoy Women and Children's Hospital.

The International Association for the Study of Pain has recently been working with the World Health Organization (WHO) to increase global awareness of poorly relieved acute pain and to highlight the burden of chronic noncancer pain. On October 11, 2004, the WHO and the European Federation of the International Association for the Study of Pain Chapters cosponsored a Global Day Against Pain urging the need for pain relief to be seen as a human right and asking governments to recognize unrelieved pain as a pressing problem. Notably absent from the press releases was any statement expressing the need to improve the lot of millions of women throughout the world who give birth in intense pain with no offer of relief.

The first lesson concerns the consequences of undertreating labour pain. As I researched this editorial, I learned that the fail- ure to address the need for prenatal education in methods of pain relief during labour had led to serious and surprising consequences in Turkey throughout the past decade. According to Yildirim and Sahin, the majority of pregnant women there do not receive any antenatal education, and for the 30\% who do, pain control is not discussed. To add further to the stress of the experience, all women in labour are observed together in the same room without a family member present until cervical dilation is complete. Do Turkish women fear labour? Absolutely! Anxiety about labour pain is so great that women who could afford it would pay their obstetrician to perform a cesarean section before the contractions began. In some urban hospitals, the cesarean section rate approached 75\% (Dr Medge Owen, Department of Anesthesiology, Wake-Forest University, Winston-Salem, North Carolina, personal communication). Due to a lack of specialized training of anesthesiologists, cesarean sections are mostly performed under general anesthesia, which carries more risk to the mother and neonate than regional anesthesia. This partially explains why the maternal mortality rate in Turkey has grown to one of the highest in Europe and is 15 times greater than that for the United States (Dr Medge Owen, Department of Anesthesiology, Wake-Forest University, Winston-Salem, North Carolina, personal communication).

To be fair, these problems are not unique to Turkey. A nationwide survey of pain relief in childbirth in neighboring Hungary published in 1997 found that no analgesia was offered in $81 \%$ of vaginal deliveries and epidural analgesia was used in fewer than $5 \%$ of vaginal deliveries (1).

What are the potential consequences of severe labour pain aside from the alarming developments described in Turkey? Intrapartum pain and anxiety are associated with high levels of circulating maternal epinephrine which may, in turn, lead to abnormal fetal heart rate patterns and dyscoordinate labour. It has also been suggested that there may be longerterm benefits of pain relief in labour, including reduced likelihood of postpartum depression, improved self-esteem, increased confidence in mothering and more successful establishment of breastfeeding (2).

The second lesson confirms current knowledge about pain. The complex experience of pain includes not only pain intensity but also fear, unpleasantness and anticipation of pain. Addressing fear and unpleasantness can reduce overall suffering. The influence of fear and anxiety on acute and chronic pain states is familiar to all clinicians. In chronic pain patients, there 
is compelling, cognitive-behavioural evidence suggesting that fear and anxiety-sensitivity affect pain-related disability (3). Thanks to functional magnetic resonance imaging, the distinction between anxiety related to the anticipation of acute pain and the perception of the pain stimulus itself can be explored. Ploghaus et al (4) demonstrated that expectation of acute thermal pain activated sites in the brain which were close to, but distinct from, locations mediating pain perception. The authors speculated that selective manipulations of activity in the anterior medial frontal cortex may offer therapeutic possibilities for treating pain. Prenatal education and the promise that one will be supported during labour do just that, although perhaps not in the high-tech fashion these writers envisioned.

Historically, it has been the custom for women to support other women during labour. However, since the 1950s it has become the norm in many countries for women to give birth in a hospital rather than at home. Concern about the dehumanization of women's birth experience led to research into the effects of continuous labour support. In 2003, the Cochrane Collaborative Review of Continuous Support for Women during Childbirth published a review (5) of 15 trials involving over 12,000 women. Those mothers who had continuous intrapartum support were less likely to use intrapartum analgesics, experience operative birth or report dissatisfaction with their childbirth process. The $\mathrm{WHO}$ has been promoting intrapartum support through its Better Birth Initiatives in China and several African countries. Uruguay actually passed a law decreeing that all women have the right to companionship in labour (5).

The third lesson is that satisfaction with the birth experience does not rely on the degree of pain relief but rather on the quality of the relationship with the caregiver and involvement in decision-making. In Yildirim and Sahin's article, even women in the experimental study group experienced intense pain in the latter stages of labour. However, positive feelings about the labour and delivery room experience were generally high in the experimental group. A systematic review (6) examining factors associated with women's satisfaction with childbirth found that four factors predominate: amount of support from caregivers, quality of relationships with caregivers, involvement with decision-making and having experiences that exceeded expectations.

Now that patient satisfaction is being increasingly monitored as an indicator of quality of care in chronic pain clinics, similar trends are being noted. It is not necessarily reduction in pain scores but, rather, respect from the caregiver and patient participation in decision-making that produce satisfaction (7, unpublished data).

The last lesson is one which we in the western world are well aware of, but it still bears repeating. Workable solutions to health care problems in developing countries require sensitivity to the culture and economic resources of the country.

Although controversies exist as to the effects of epidural analgesia on labour and the neonate, its undeniable efficacy has made it the most widely used method of pain relief in all mid- to large-size birthing centres in North America. However, to offer epidural analgesia on demand in an environment where 50 women give birth in $24 \mathrm{~h}$ is clearly untenable and would create more problems than it solved. Kybele, a nonprofit organization devoted to improving safe childbirth in developing countries, has targeted Turkey as a country that not only possesses an infrastructure of well-supplied hospitals but is also receptive to change and specifically interested in increasing physician training in obstetric anesthesia. For further information on this organization and the progress it has made in reforming attitudes to childbirth in Turkey, the reader is encouraged to visit the Web site affiliated with Wake-Forest University < www.wfubmc.edu/anesthesia/Kybele>.

Currently, one of the most pressing global pain problems, as highlighted by the Global Day Against Pain, is the suffering from HIV-related pain. This is an enormous challenge that threatens to be overwhelming. The only chance of meeting this enormous challenge lies in initially targeting countries with an appropriate infrastructure, as Kybele has done for childbirth pain. The focus needs to be on locally attainable goals such as more effective topical analgesia or tricyclic antidepressants. Proposed solutions in the western world, such as increasing the availability of opioids, must be carefully considered to avoid the creation of further problems of illicit drug trade and/or addiction in poverty-stricken regions.

Experience in Turkey demonstrated that failure to address the problem of alleviating labour pain led to serious safety concerns. These are now being successfully rectified through the joint efforts of Turkish health care workers, government officials and foreign-trained obstetric anesthesiologists under the auspices of the Society of Obstetric Anesthesiology and Perinatology. Locally based research efforts such as the trial by Yildirim and Sahin are promoting changes in attitudes. Hopefully, the lessons learned can be translated to improve the management of acute and chronic pain conditions prevalent in other developing countries.

\section{REFERENCES}

1. Beke A, Takacs G, Sziller I, Fedak L, Papp Z. Obstetric anaesthesia in Hungary. Int J Obstet Anesth 1997;6:235-8.

2. Hofmeyr GJ, Nikodem VC, Wolman WL, Chalmers BE, Kramer T. Companionship to modify the clinical birth environment: Effects on progress and perceptions of labour, and breastfeeding. Br J Obstet Gynaecol 1991;98:756-64

3. Asmundson GJ, Norton PJ, Norton GR. Beyond pain: The role of fear and avoidance in chronicity. Clin Psychol Rev 1999;19:97-119.

4. Ploghaus A, Tracey I, Gati JS, et al. Dissociating pain from its anticipation in the human brain. Science 1999;284:1979-81.

5. Hodnett ED, Gates S, Hofmeyr GJ, Sakala C. Continuous support for women during childbirth (Cochrane Review). In: The Cochrane Library, Issue 4, 2004. Chichester, UK: John Wiley \& Sons, Ltd.

6. Shapiro A, Fredman B, Zohar E, Olsfanger D, Jedeikin R. Delivery room analgesia: An analysis of maternal satisfaction. Int J Obstet Anesth 1998;7:226-30.

7. Horrill K, Morley-Forster PK. The role of the nurse-practitioner in an outpatient chronic pain clinic. 2nd Joint Scientific Meeting of the American Pain Society and the Canadian Pain Society. Vancouver, May 6 to 9, 2004. (Abst) 


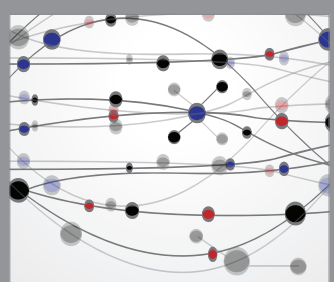

The Scientific World Journal
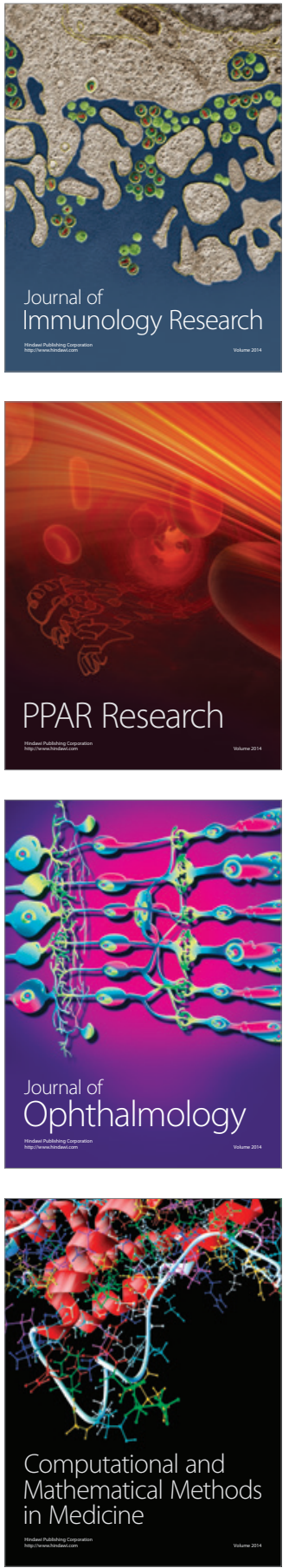

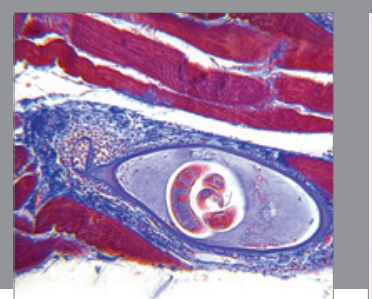

Gastroenterology Research and Practice

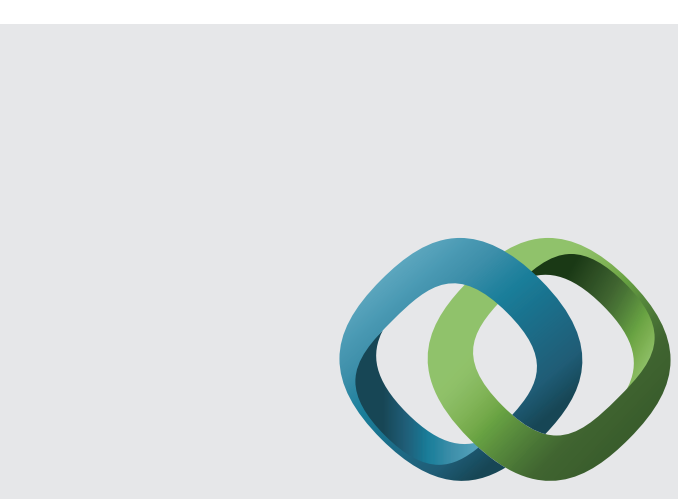

\section{Hindawi}

Submit your manuscripts at

http://www.hindawi.com
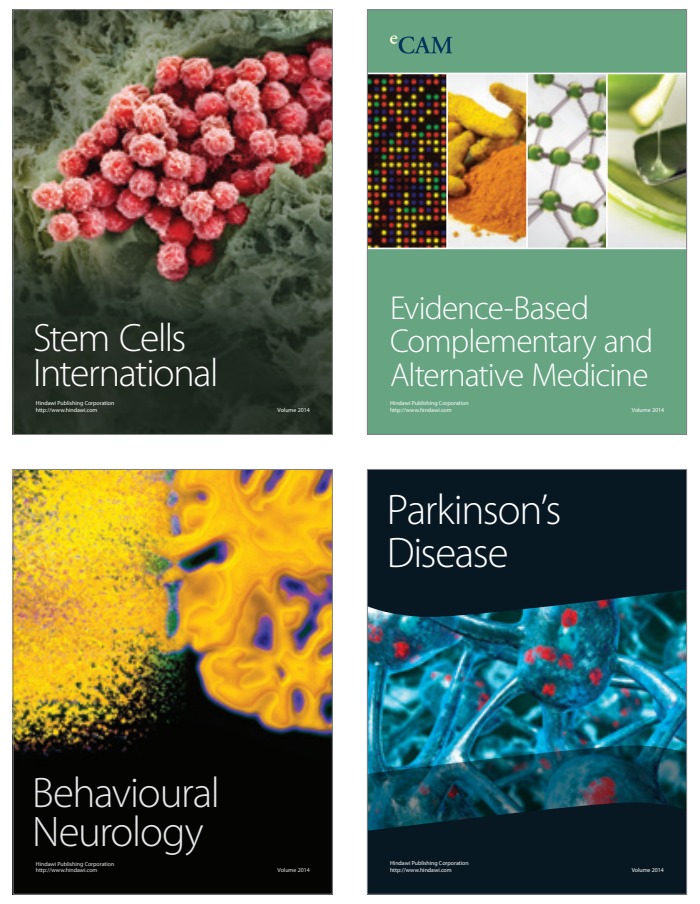
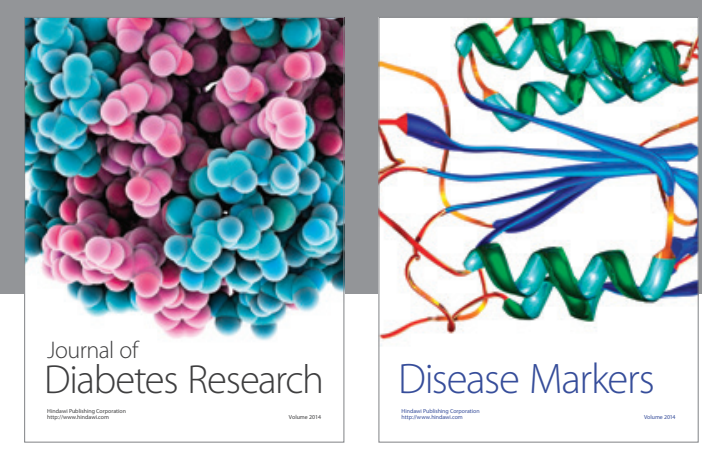

Disease Markers
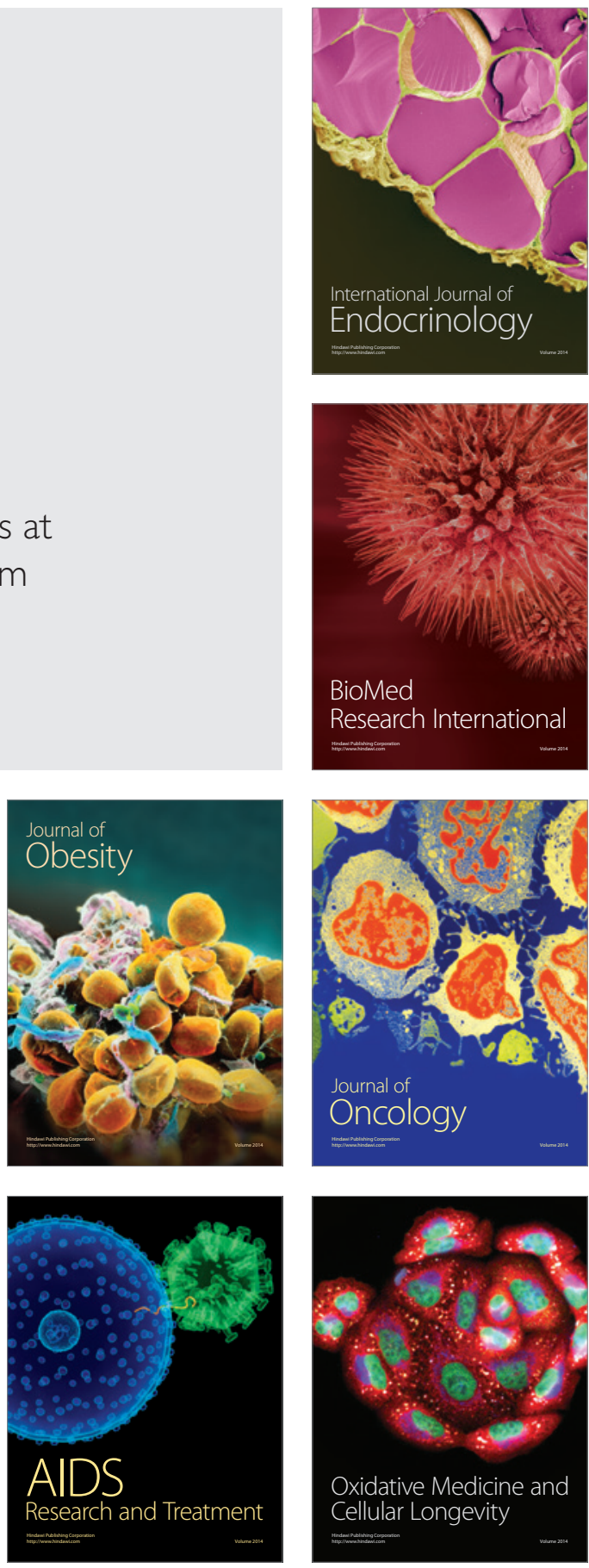